\title{
INVESTIGATION OF THE INFLUENCE OF DISPERSION DISTORTIONS OF WIDEBAND SIGNALS ON THE QUALITY OF THEIR DELAY AND FREQUENCY SHIFT ESTIMATION
}

\author{
Evgeniy M. Lobov, Nikolai A. Kandaurov, Elizaveta O. Lobova, Vadislav I. Lipatkin, \\ Moscow Technical University of Communications and Informatics, Moscow, Russia, \\ lobov@srd.mtuci.ru
}

DOI: |0.36724/2664-066X-202|-7-I-I|I-I6

\begin{abstract}
The article investigates the quality of algorithms for esimate the main parameters of wideband signals in the problem of over-the-horizon radar under conditions of dispersion distortions in the Earth's ionosphere. The boundary values of the variances of the estimates of propagation delay, frequency shift and phase, wideband phase-shift keyed signals are investigated. It is shown that dispersion distortions of the radar signal lead to a correlation between the estimates of the delay and frequency shift. The curves of the dependence of the frequency-time ratio depending on the length of the used pseudo-random sequence and the degree of dispersion distortions are obtained. Analytical curves of the Cramer-Rao lower bounds are constructed for the variances of estimates and the values of the correlation coefficients of the estimated parameters in the presence of dispersion distortions, which are confirmed by the results of simulation.
\end{abstract}

The reported study was funded by RFBR, project number 19-29-06055.

KEYWORDS: broadband signal, ionosphere channel, frequency dispersion, joint estimation, frequency-time ratio, quality of the estimation. 


\section{Introduction}

Over-the-horizon radar systems operate in the shortwave range and are designed to detect targets at distances of the order of several thousand kilometers. The quantitative indicators of the quality of the radar system can be improved through the use of wideband signals. However, wideband signals are sensitive to the frequency dispersion of the ionospheric channel, which is the reason for a significant decrease in the quality of their detection [1-13]. In this paper, we study the influence of the frequency dispersion of the ionosphere on the quality of the joint estimate of the Doppler frequency shift, delay, and initial phase.

\section{The signal and the channel model}

The complex signal at the output of the quadrature demodulator is presented as a mixture of the information signal, distorted by the frequency dispersion of the ionospheric channel, and white Gaussian noise

$$
\begin{aligned}
& \dot{x}\left(t, \varphi, \tau, f_{d}, s\right)=e^{-j \varphi} e^{j 2 \pi f_{d}(t-\tau)} \dot{x}(t-\tau, s)+\dot{n}(t), \\
& t=-T_{s} / 2 \div T_{s} / 2
\end{aligned}
$$

where $\dot{x}(t, s)=\dot{x}(t) * \dot{h}(t, s)$ - complex envelope of the useful signal distorted by the ionospheric channel, $\dot{h}(t, s)$ - complex envelope of the impulse response (IR) of the ionospheric channel, $\dot{x}(t)$ - complex envelope of the useful signal undistorted, $f_{d}-$ the Doppler frequency shift, $\tau$-delay, $S$ - the slope of the dispersion characteristic which characterize the effect of frequency dispersion on the signal, $\varphi$ - an unknown phase shift, $\dot{n}(t)-$ complex envelope of white Gaussian noise with zero mean and variance $\sigma_{u}^{2}, T_{s}$ - signal duration.

As a model of a channel with frequency dispersion, a channel model with a linear dependence of the signal group delay on the central frequency (linear dispersion characteristic with slope s) [1] is adopted. The transfer function of the ionospheric channel in the absence of multipath signal propagation can be written in the form

$$
H(j 2 \pi f)=\mathrm{e}^{-j \pi s f^{2}}, f \in[-\Delta f / 2 ; \Delta f / 2]
$$

where $\Delta f-$ width of the transfer function of the ionospheric channel, $S$ - the slope of the dispersion characteristic. The phase-frequency response of the channel is described by a quadratic dependence on frequency, and the group propagation delay is described by a linear one with a slope $S \mu \mathrm{s} / \mathrm{MHz}$. We will assume that the slope of the dispersion characteristic $s \mu \mathrm{s} / \mathrm{MHz}$ is known at the receiving side, while the delay $\tau$, the Doppler frequency shift $f_{d}$ and signal phase $\varphi$ - unknown non-energy parameters that need to be estimated.

To estimate the parameters under study, it is proposed to apply the method of the maximum likelihood (ML). When using the ML method, the decision about the prox- imity of one or another estimate value to the true value of the parameter being determined is made according to the following rule (in the case of coherent receiving) [14]:

$$
\lambda=\max \left(\operatorname{Re}\left[\int_{-T_{s} / 2}^{T_{s} / 2} \dot{y}(t) \tilde{u}^{*}\left(t, s, \hat{f}_{d}, \hat{\tau}, \hat{\varphi}\right) d t\right]\right),
$$

$\dot{y}(t)$ - additive mixture of useful signal quadratures and white Gaussian noise in continuous time, determined by (1), $\tilde{\dot{u}}\left(t, s, \hat{f}_{d}, \hat{\tau}, \hat{\varphi}\right)$ - ref rence signal generated on the basis of a priori information about the useful signal, $\hat{f}_{d}-$ the Doppler shift estimate, $\hat{\tau}$ - delay estimate, $\hat{\varphi}$ - estimate an initial phase, * - complex conjugate symbol.

To analyze the effect of dispersion distortions on the quality of the estimate, we define the variance and crosscorrelation $\hat{\tau}, \hat{f}_{d}$ and $\hat{\varphi}$.

\section{The estimation variance lower bound}

The correlation of the estimate of unknown parameters in the first approximation [14]

$$
K_{i k}\left(\mathbf{l}_{m}, \mathbf{l}\right)=\left.\frac{A_{k i}}{\rho^{2} \Omega}\right|_{\mathbf{l}_{m} \rightarrow \mathbf{1}},
$$

where $\Omega$ - determinant of a matrix $W$, consisting of a set of partial derivatives $-\frac{\partial^{2} S}{\partial l_{i} \partial l_{j}}, S-$ real part of the signal component of the correlation integral (3), $A_{k i}$ - algebraic complements of a matrix $W, \mathbf{l}_{m}$ - vector of parameter estimates, $\mathbf{l}$ - vector of estimated parameters, $i=1 \div K$, $k=1 \div K, K-$ the number of estimated parameters (in the case considered in the article $K=3$ ), $\rho_{S N R}^{2}=2 E_{s} / N_{0}-$ signal-to-noise ratio (SNR), $N_{0}-$ power spectral density (PSD) of white Gaussian noise. For coinciding indices $i$ and $k$ (4) corresponds to the variances of estimates of the parameters under study.

Having carried out all the necessary mathematical transformations and calculations over expression(4), we obtain formulas that allow us to calculate the variances and cross-correlations of the estimates of the studied parameters in the first approximation:

$$
\begin{aligned}
& D\left(\hat{f}_{d}\right)=\frac{1}{4 \pi^{2} \rho_{S N R}^{2} T_{e f f}^{2}\left(1-\rho_{\tau f_{d}}^{2}\right)}, \\
& D(\hat{\tau})=\frac{1}{4 \pi^{2} \rho_{S N R}^{2} F_{e f f}^{2}\left(1-\rho_{\tau f_{d}}^{2}\right)}, \\
& D(\hat{\varphi})=\frac{1-\rho_{\tau f_{d}}^{2}+\frac{f_{d}^{2}}{F_{e f f}^{2}}}{4 \pi^{2} \rho_{S N R}^{2}\left(1-\rho_{\tau f_{d}}^{2}\right)},
\end{aligned}
$$




$$
\begin{aligned}
& K\left(\hat{f}_{d}, \hat{\tau}\right)=\frac{1}{4 \pi^{2} \rho_{S N R}^{2} T_{e f f} F_{e f f}\left(1-\rho_{\tau f_{d}}^{2}\right)}, \\
& K\left(\hat{f}_{d}, \hat{\varphi}\right)=\frac{-f_{0} \rho_{\tau f_{d}}}{2 \pi \rho_{S N R}^{2} T_{e f f} F_{e f f}\left(1-\rho_{\tau f_{d}}^{2}\right)}, \\
& K(\hat{\tau}, \hat{\varphi})=\frac{-f_{0}}{2 \pi \rho_{S N R}^{2} F_{e f f}^{2}\left(1-\rho_{\tau f_{d}}^{2}\right)},
\end{aligned}
$$

where $T_{\text {eff }}=\frac{\int_{-\infty}^{\infty}(t-\tau)|\dot{x}(t-\tau)| d t}{\int_{-\infty}^{\infty}|\dot{x}(t-\tau)| d t}-$ ef ctive signal duration, $F_{\text {eff }}=\frac{\int_{-\infty}^{\infty} f^{2}|\dot{X}(j 2 \pi f)|^{2} d f}{\int_{-\infty}^{\infty}|\dot{X}(j 2 \pi f)|^{2} d f}-$ effective signal ndwidth, $\dot{X}(j 2 \pi f)-$ complex signal spectrum $\dot{x}(t)$, $\rho_{\tau f_{d}}=\frac{\int_{-\infty}^{\infty} t f_{s}(t)|\dot{x}(t)|^{2} d t}{F_{e f f} T_{e f f} \int_{-\infty}^{\infty}|\dot{x}(t)|^{2} d t}-\mathrm{t}$ e-frequency ratio, $f_{s}(t)=\frac{d \gamma(t)}{2 \pi d t}-$ frequency modulation law signal $\dot{x}(t)$, $\gamma(t)$-argument $\dot{x}(t), \dot{x}(t)=|\dot{x}(t)| e^{j \gamma(t)}$.

\section{Simulation results}

To confirm the theoretical results, simulation modeling was carried out for a single rectangular pulse and a sequence of rectangular pulses with a spectral width $\Delta F=400 \mathrm{kHz}$. Doppler frequency shift has been set $f_{d}$ - $0 \mathrm{kHz}$, delay $\tau-0.1 \mu \mathrm{s}$, phase shift $\varphi-90$ degrees. As a result of the simulation, graphs of the dependences of the frequency-time ratio $\rho_{\tau f_{d}}$ on $\rho=\frac{\Delta F}{\Delta f_{c o h}}, \Delta f_{c o h}=\sqrt{\frac{4}{\pi s}}$ - ch nnel coherence bandwith for different types of signals.

1 shows this dependence for a single rectangular pulse and short sequences of rectangular pulses based on the Barker code of different lengths [15].

2 shows the dependence of $\rho_{\tau f_{d}}$ on $\rho$ was obtained for a sequence of rectangular pulses based on Gold sequences of different lengths which obviously has small values of $\rho_{\tau f_{d}}$.
From the dependences shown in the figures, it can be concluded that the effect of frequency dispersion increases the value of $\rho_{\tau f_{d}}$, thereby making the correlation ellipse ever narrower, which negatively affects the quality of the joint measurement of the delay and of the Doppler frequency shift.

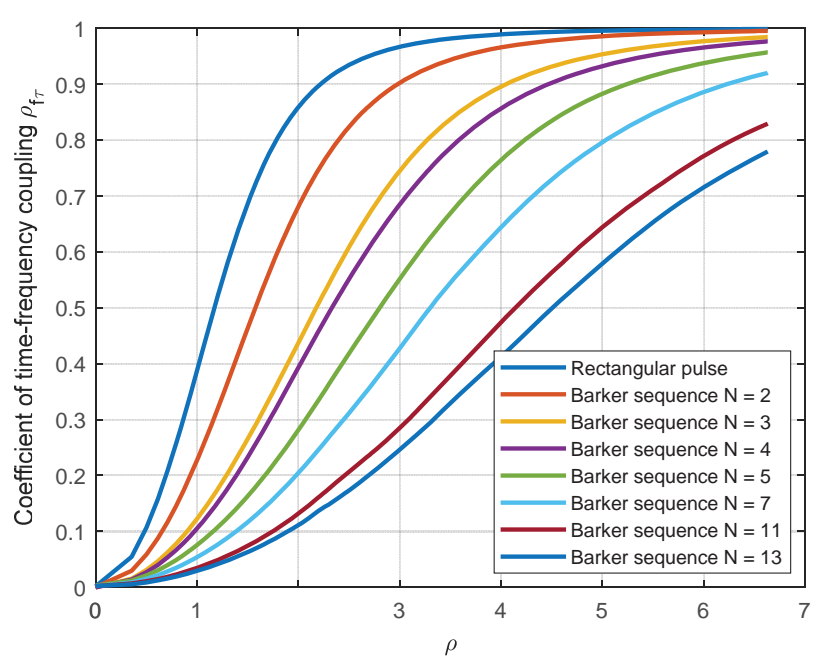

Figure 1. Time-frequency ratio versus $\rho$

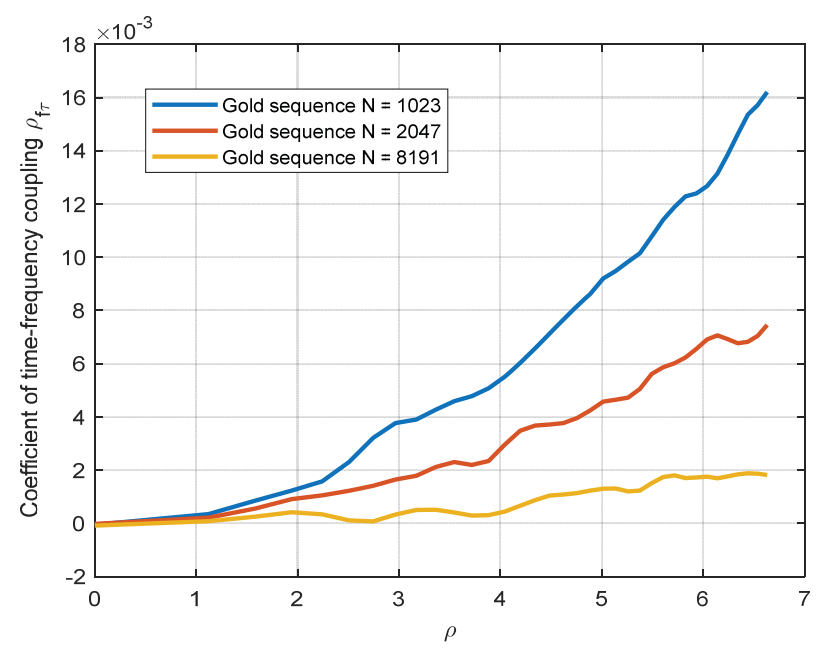

Figure 2. Time-frequency ratio versus $\rho$ for long sequences

Also, during the simulation, the values of the parameters $\hat{f}_{d}, \hat{\tau}$ and $\hat{\varphi}$ were obtained, the standard deviation and cross-correlation of estimates for different SNR values were calculated. The simulation was performed under $s=70 \mu \mathrm{s} / \mathrm{MHz}$ for a sequence of rectangular pulses of length $N=7$.

Figure 3 - 11 show the dependences of theoretical and sample standard deviation of the $\hat{f}_{d}, \hat{\tau}$ and $\hat{\varphi}$ estimates, and their cross-correlation functions and coefficient of cross-correlation versus SNR. 


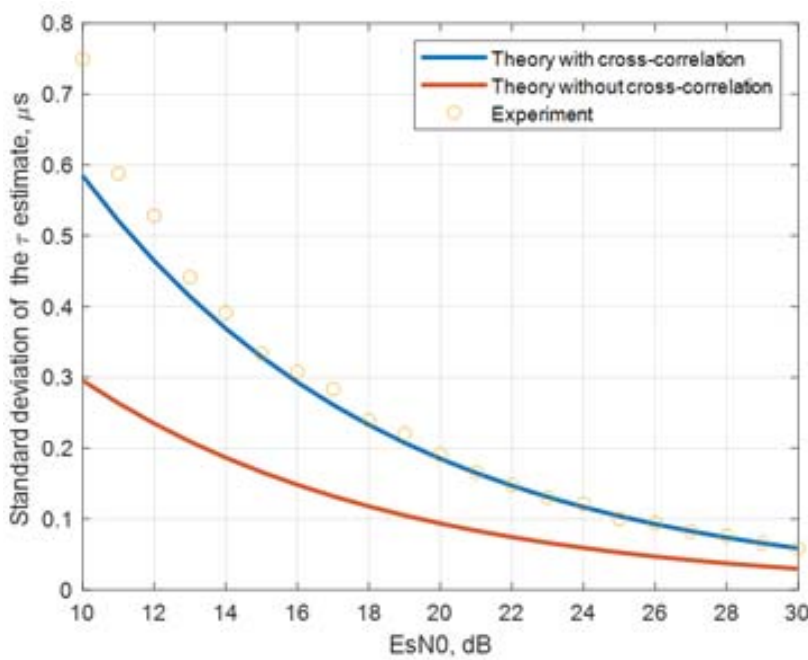

Figure 3. Standard deviation of the $\tau$ estimate versus SNR

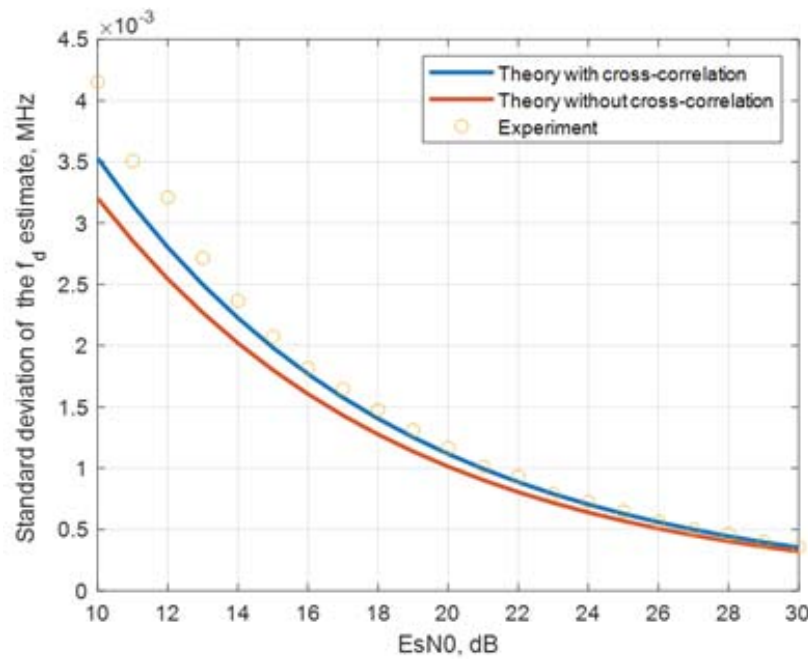

Figure 4. Standard deviation of the $f_{d}$ estimate versus SNR

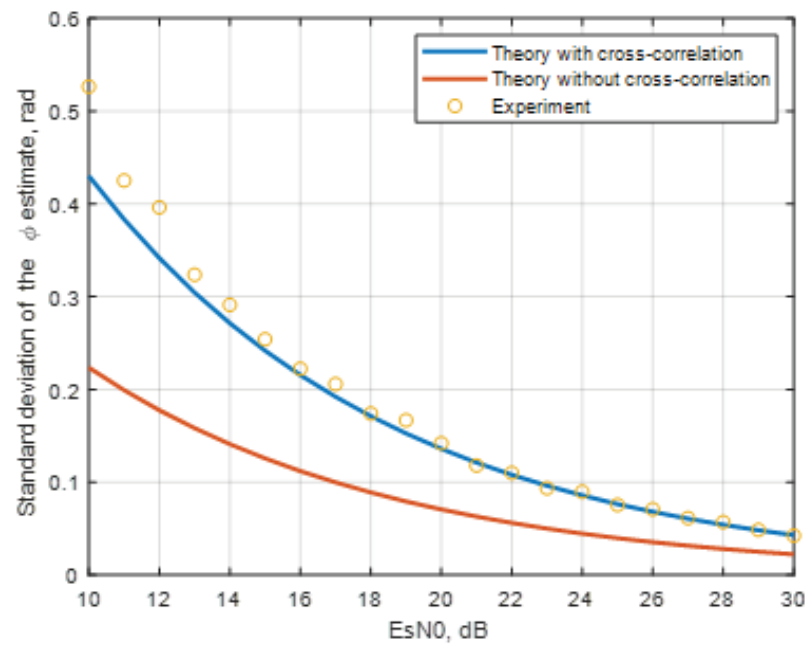

Figure 5. Standard deviation of the $\varphi$ estimate versus SNR

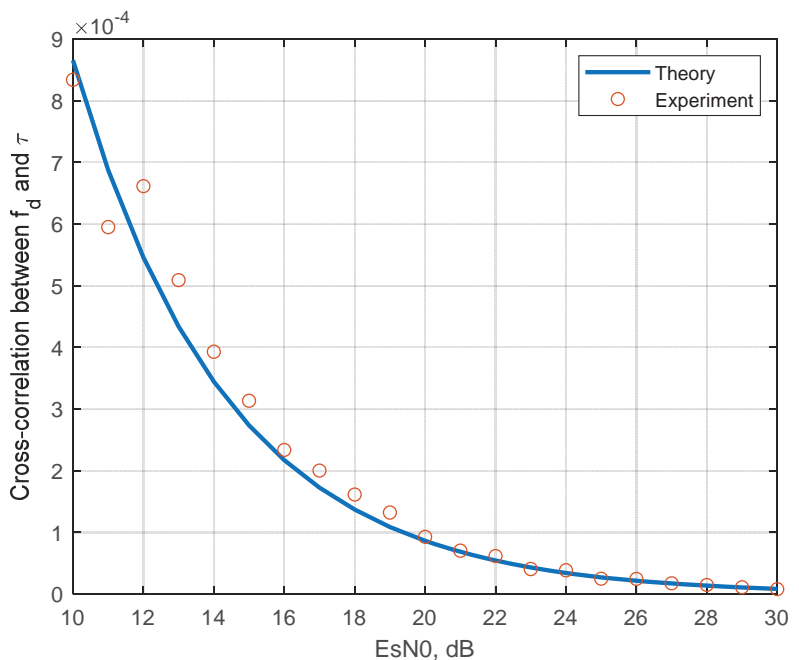

Figure 6. Cross-correlation between $f_{d}$ and $\tau$ versus SNR

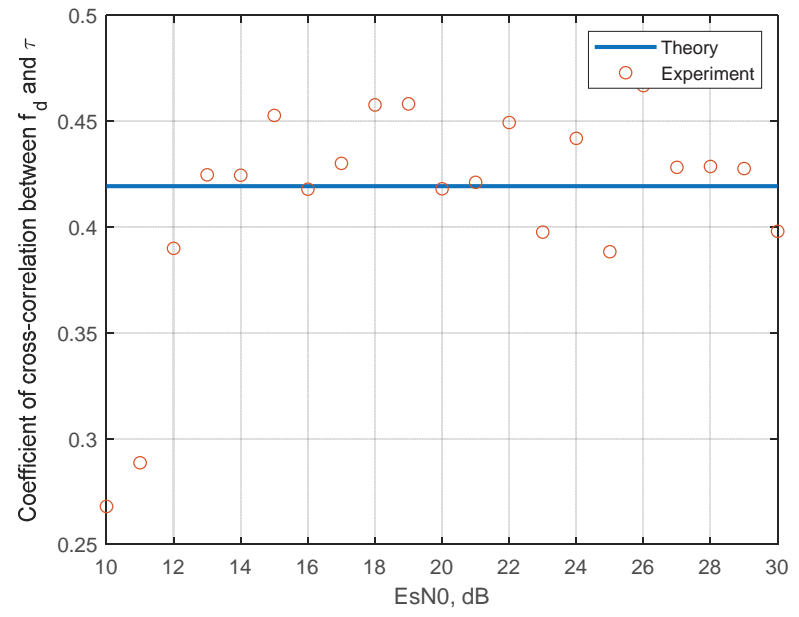

Figure 7. Coefficient of cross-correlation between $f_{d}$ and $\tau$ versus SNR

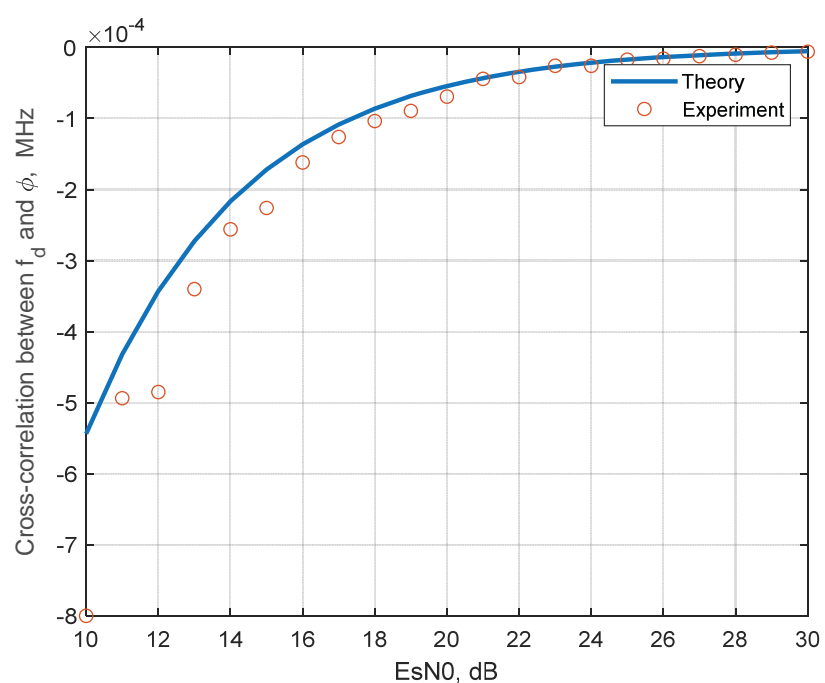

Figure 8. Cross-correlation between $f_{d}$ and $\varphi$ versus SNR 


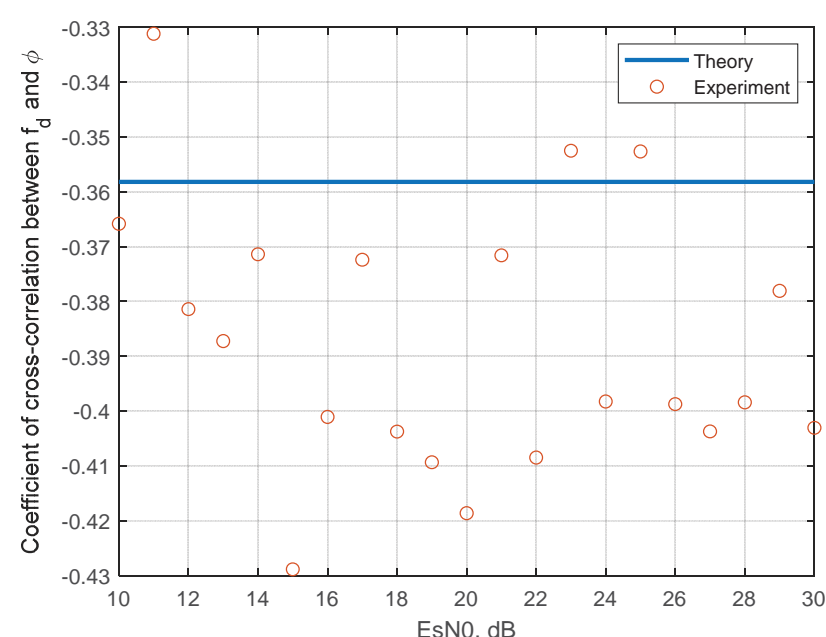

Figure 9. Coefficient of cross-correlation between $f_{d}$ and $\varphi$ versus SNR

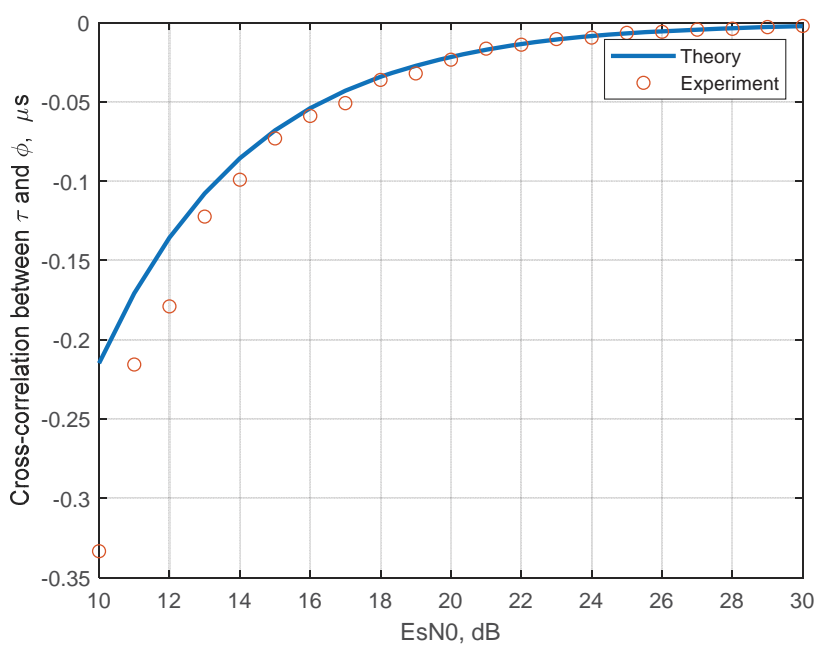

Figure 10. Cross-correlation between $\tau$ and $\varphi$ versus SNR

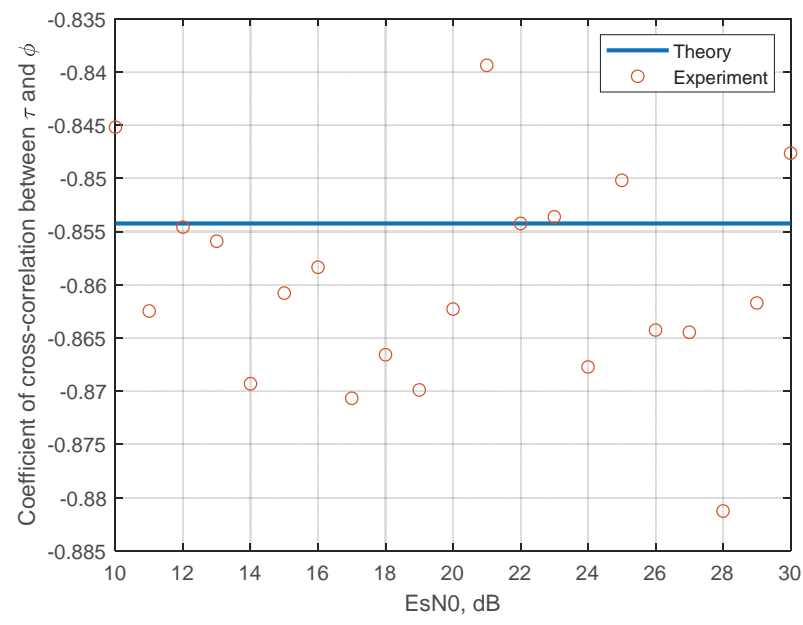

Figure 11. Coefficient of cross-correlation between $\tau$ and $\varphi$ versus SNR
The above analysis of dependencies can draw the following conclusions:

1. The figures show that the standard deviation of the estimates of all the parameters studied in the article during their joint estimation is greater than if all parameters were estimated separately from each other. This is due to the appearance of cross-correlations between estimates.

2. The results of theoretical studies and simulation modeling showed the presence of a cross-correlation between the investigated estimates: $K\left(\hat{f}_{d}, \hat{\tau}\right) \neq 0$, $K\left(\hat{f}_{d}, \hat{\varphi}\right) \neq 0, \quad K(\hat{\tau}, \hat{\varphi}) \neq 0$. Cross-correlation coefficients in the figures $7,9,11$ shows that the crosscorrelation between the estimates $\hat{f}_{d}$ and $\hat{\tau}, \hat{f}_{d}$ and $\hat{\varphi}, \hat{\tau}$ and $\hat{\varphi}$ is significant, therefore, between the estimates of these parameters there is an obvious correlation, which affects the quality of their joint estimate.

\section{Conclusion}

The article investigated the effect of frequency dispersion on the quality of the joint estimates of Doppler frequency shift, delay and initial phase. In the present work, theoretical expressions are obtained for the variances and cross-correlations of estimates of the indicated unknown parameters for a signal with an arbitrary spectrum. It is shown that the effect of frequency dispersion increases the value of the time-frequency coupling coefficient, thereby making the correlation dependences between the investigated estimates stronger, which negatively affects the quality of the joint measurement of the delay and the Doppler frequency shift.

Acknowledgment. The reported study was funded by RFBR, project number 19-29-06055.

\section{References}

1. D.V. Ivanov, Methods and mathematical models for study of the propagation of decameter complex signals and correction its dispersion distortions. MarSTU, Yoshkar-Ola, 2006.

2. E.M. Lobov, I.S. Kosilov, "Calculation of noise immunity of broadband ionospheric radio links using noise-like signals based on prediction data," T-Comm, 2011, vol. 5, no. 11, pp. 68-70.

3. E.M. Lobov, E.O. Lobova, N.A. Kandaurov, "Optimum tracking dispersion distortion compensator," Telecommunications, 2018, no.5. P. 85-89.

4. E.O. Lobova, N.A. Kandaurov, "Experimental Results of Dispersion Distortion Compensation of Wideband Signals with a Device Based on a Digital Filter Bank," 2019 Systems of Signals Generating and Processing in the Field of on Board Communications, SOSG 2019, Moscow, 20-21 March 2019, 8 p. (DOI: 10.1109/SOSG.2019.8706758). 
5. E.O. Lobova, E.M. Lobov, B.A. Elsukov, "Broadband Signals Dispersion Distortions Compensator Based on the Digital Filters Bank," Reports of the 20th International Conference "Digital Signal Processing and its Application - DSPA-2018", Moscow, Russia, vol. 2. P. 487-491.

6. E.O. Lobova, E.M. Lobov, B.A. Elsukov, "Wideband signals dispersion distortion compensator based on digital filter banks," 2018 Systems of signals generating and processing in the field of on board communications, Moscow, 14-15 March 2018, 4 p. (DOI: 10.1109/SOSG.2018.8350615)

7. E.O. Lobova, "Investigation of the quality of algorithms for estimating the slope of the ionospheric channel dispersion characteristics," Telecommunications, 2019, no. 5. P. 48-43.

8. E.O. Lobova, "Determining Channels Number in the Digital Filter Bank for the Wideband Signals Dispersion Distortion Compensation," 2020 Systems of Signals Generating and Processing in the Field of on Board Communications, 19-20 March 2020, Moscow, 6 p.

9. V.I. Lipatkin, E.O. Lobova, N.A. Kandaurov, "Wideband Signals Dispersion Distortions Optimum Tracking Compensator Based On Digital Filter Banks Using Farrow Filters,' 2020 Systems of Signals Generating and Processing in the Field of on Board Communications, 19-20 March 2020, Moscow, 6 p.

10. V.I. Lipatkin, E.O. Lobova "Broadband Noise-like Signal Parameters Joint Estimation Quality with Dispersion
Distortions in the Ionospheric Channel", 2020 Systems of Signal Synchronization, Generating and Processing in Telecommunications, SYNCHROINFO 2020, Svetlogorsk, 1-3 July 2020, 6p. (DOI: 10.1109/SYNCHROINFO49631.2020.9166082)

11. E.M. Lobov, E.O. Smerdova, N.A. Kandaurov, I.S. Kosilov, B.A. Elsukov, "Optimum estimation and filtering of the ionospheric channel dispersion characteristics slope algorithms," Systems of signal synchronization, generating and processing in telecommunications, SINKHROINFO 2017, Kazan, 3-4 July 2017, Kazan, 7p. (DOI: 10.1109/SINKHROINFO.2017.7997537)

12. S.S. Adjemov, E.M. Lobov, N.A. Kandaurov, E.O. Lobova, "Methods and algorithms of broadband HF signals dispersion distortion compensation," 2019 Systems of Signal Synchronization, Generating and Processing in Telecommunications, SYNCHROINFO 2019, Yaroslavl, 1-3 July 2019, 9p. (DOI: $10.1109 /$ SYNCHROINFO.2019.8814074)

13. E.M. Lobov, E.O. Smerdova, "Investigation of the quality of algorithms for estimating the slope of the ionospheric channel dispersion characteristics," Telecommunications, 2017, no. 6. P. 28-31.

14. E.I. Kulikov, A.P. Trifonov. Signal parameters estimation in the noise. Moscow, Soviet Radio, 1978. 296 p.

15. L.E. Varakin. Communication systems with noise-like signals. Moscow, Radio and Telecommunication, 1985. 384 p. 